\title{
2D-DOA Estimation of Noncircular Signals for Uniform Rectangular Array via Noncircular-ESPRIT
}

\author{
Weihua Lv $^{1,2}$, Wang Zheng ${ }^{1}$, Xiaofei Zhang ${ }^{1,3}$, Licen Zhang ${ }^{1}$ \\ 1. College of Electronic and Information Engineering, Nanjing University of Aeronautics and \\ Astronautics, Nanjing, China, 210016 \\ 2. Luoyang Optoelectro technology development center, Luoyang, China, 471000 \\ 3. National Mobile Communications Research Laboratory, Southeast University, China, 210096 \\ Email: fei_zxf@163.com
}

Keywords: uniform rectangular array, two-dimensional direction of arrival (2D-DOA), noncircular signals, noncircular-ESPRIT.

\begin{abstract}
In this paper, we propose the noncircular estimation signal parameters via rotational invariance techniques (NC-ESPRIT) algorithm as a two-dimension direction of arrival (2D-DOA) estimation algorithm for uniform rectangular array. Compared to conventional circular ESPRIT algorithm, the proposed algorithm exploits the property of noncircular signals through data expansion, so that the array aperture is doubled. Therefore, the angle estimation performance is superior to that of the circular ESPRIT algorithm. Moreover the proposed algorithm has no spectral peak searching and the two-dimension angle estimates can be paired automatically. Simulation results illustrate the effectiveness and improvement of the proposed algorithm.
\end{abstract}

\section{Introduction}

Direction of arrival (DOA) estimation has been a central issue in array signal processing and crucial part of communication, radar, sonar and radio telescope [1-4]. The ESPRIT algorithm requires no spectral peak searching. Actually, noncircular signals are frequently encountered in digital communications [5], e.g., binary phase shift keying (BPSK) and amplitude modulation (AM) signals. In the field of array signal processing, the noncircular signal has attracted a great attention due to the performance improvement brought by its noncircular property [6-9]. In this paper, we connect the ESPRIT method and the property of noncircular signal, and propose a noncircular ESPRIT (NC-ESPRIT) algorithm to estimate the 2D-DOA of noncircular signals for uniform rectangular array. Our algorithm can estimate 2D-DOA with automatic pairing and needs no peak searching, which has better performance than circular ESPRIT.

The reminder of this paper is structured as follows. Section II describes the data model. Section III introduces the proposed NC-ESPRIT algorithm and the complexity analysis. Section IV presents numerical simulations to illustrate the performance of our method, and the conclusions are drawn in Section V.

\section{Data model}

Consider a uniform rectangular array with $M \times N$ antenna elements, where $M$ and $N$ are the numbers of elements along $x$-axis and $y$-axis, respectively. The distance between the two adjacent elements is $d$. Assume that there are $K$ uncorrelated signal sources impinging on the array, each of which has the elevation angle of $\theta_{k}$ and the azimuth angle of $\phi_{k}$. The received signal of the rectangular array can be expressed as

$$
\mathbf{X}=\left[\begin{array}{c}
\mathbf{X}_{1} \\
\mathbf{X}_{2} \\
\vdots \\
\mathbf{X}_{N}
\end{array}\right]=\left[\begin{array}{c}
\mathbf{A}_{x} D_{1}\left(\mathbf{A}_{y}\right) \\
\mathbf{A}_{x} D_{2}\left(\mathbf{A}_{y}\right) \\
\vdots \\
\mathbf{A}_{x} D_{N}\left(\mathbf{A}_{y}\right)
\end{array}\right] \mathbf{S}+\left[\begin{array}{c}
\mathbf{N}_{1} \\
\mathbf{N}_{2} \\
\vdots \\
\mathbf{N}_{N}
\end{array}\right]
$$


where $\mathbf{A}_{x}=\left[\mathbf{a}_{x}\left(\phi_{1}, \theta_{1}\right), \mathbf{a}_{x}\left(\phi_{2}, \theta_{2}\right), \cdots, \mathbf{a}_{x}\left(\phi_{K}, \theta_{K}\right)\right] \in \mathbf{C}^{M \times K}$ is the steering matrix of the $M$ elements in $x$-axis, $\mathbf{A}_{y}=\left[\mathbf{a}_{y}\left(\phi_{1}, \theta_{1}\right), \mathbf{a}_{y}\left(\phi_{2}, \theta_{2}\right), \cdots, \mathbf{a}_{y}\left(\phi_{K}, \theta_{K}\right)\right] \in \mathbf{C}^{N \times K}$ is the steering matrix of the $N$ elements in $y$-axis, $\mathbf{a}_{x}\left(\phi_{k}, \theta_{k}\right)=\left[1, e^{-j 2 \pi d \sin \theta_{k} \cos \phi_{k} / \lambda}, \cdots, e^{-j 2 \pi(M-1) d \sin \theta_{k} \cos \phi_{k} / \lambda}\right]^{T} \in \mathbf{C}^{M \times 1}$ is the array steering vector of the Kth signal source, and $\lambda$ is the wavelength. Moreover, $\mathbf{N}=[\mathbf{n}(1), \mathbf{n}(2), \cdots, \mathbf{n}(L)] \in \mathbf{C}^{M N \times L}$ is the received noise matrix. $\mathbf{S}=\boldsymbol{\psi} \boldsymbol{S}_{0}=[\mathbf{s}(1), \mathbf{s}(2), \cdots, \mathbf{s}(L)] \in \mathbf{C}^{K \times L}$, where $\boldsymbol{\psi}=\operatorname{diag}\left\{\mathrm{e}^{j \psi_{1}}, \cdots, \mathrm{e}^{j \psi_{K}}\right\}$ contains complex phase shifts on its diagonal that are different from each other. $\boldsymbol{S}_{0} \in \mathbf{R}^{K \times L}$ is a real-valued matrix.

\section{D-DOA Estimation via NC-ESPRIT}

Extend the received data matrix $\boldsymbol{X} \in \mathbf{C}^{M N \times L}$ to $\mathbf{Y}=\left[\begin{array}{c}\mathbf{X} \\ \mathbf{J} \mathbf{X}^{*}\end{array}\right] \in \mathbf{C}^{2 M N \times L}$, where $\mathbf{J}$ is the $M N \times M N$ exchange matrix with ones on its anti-diagonal and zeros elsewhere, and $\mathbf{X}^{*} \in \mathbf{C}^{M N \times L}$ stands for the conjugation of $\mathbf{X}$. The received matrix can also be denoted as

$$
\mathbf{Y}=\left[\begin{array}{c}
\mathbf{A} \mathbf{S} \\
\mathbf{J A}^{*} \mathbf{S}^{*}
\end{array}\right]+\left[\begin{array}{c}
\mathbf{N} \\
\mathbf{J} \mathbf{N}^{*}
\end{array}\right]=\left[\begin{array}{c}
\mathbf{A}_{\mathbf{x}} D_{1}\left(\mathbf{A}_{\mathbf{y}}\right) \boldsymbol{\psi} \boldsymbol{S}_{0} \\
\vdots \\
\mathbf{A}_{\mathbf{x}} D_{\mathbf{N}}\left(\mathbf{A}_{\mathbf{y}}\right) \boldsymbol{\psi} \boldsymbol{S}_{0} \\
\mathbf{A}_{\mathbf{x}} \mathbf{\Phi} D_{1}\left(\mathbf{A}_{\mathbf{y}}\right) \boldsymbol{\varphi} \boldsymbol{\psi}^{\prime} \boldsymbol{S}_{0} \\
\vdots \\
\mathbf{A}_{\mathbf{x}} \mathbf{\Phi} D_{\mathbf{N}}\left(\mathbf{A}_{\mathbf{y}}\right) \boldsymbol{\varphi} \boldsymbol{\psi}^{\prime} \boldsymbol{S}_{0}
\end{array}\right]+\tilde{\mathbf{N}}
$$

where $\boldsymbol{\Phi}=\operatorname{diag}\left\{e^{j 2 \pi d(M-1) \sin \theta_{1} \cos \phi_{1} / \lambda}, e^{j 2 \pi d(M-1) \sin \theta_{2} \cos \phi_{2} / \lambda}, \cdots, e^{j 2 \pi d(M-1) \sin \theta_{K} \cos \phi_{K} / \lambda}\right\}, \boldsymbol{\varphi}=\operatorname{diag}\left\{e^{j 2 \pi d(N-1) \sin \theta_{1} \sin \phi_{1} / \lambda}\right.$, $\left.e^{j 2 \pi d(N-1) \sin \theta_{2} \sin \phi_{2} / \lambda}, \cdots, e^{j 2 \pi d(N-1) \sin \theta_{K} \sin \phi_{K} / \lambda}\right\}, \boldsymbol{\psi}^{\prime}=J^{\prime *} \boldsymbol{\psi}^{-1}=\operatorname{diag}\left\{\mathrm{e}^{-j \psi_{K}}, \cdots, \mathrm{e}^{-j \psi_{1}}\right\}$, and $\tilde{\mathbf{N}}=\left[\begin{array}{c}\mathbf{N} \\ \mathbf{J N}^{*}\end{array}\right] \in \mathbf{C}^{2 M N \times L}$ represents the reconstructed noise matrix.

Put $\mathbf{A}_{\mathrm{x}}^{\prime}=\mathbf{A}_{\mathrm{x}} \boldsymbol{\psi}, \boldsymbol{\eta}=\boldsymbol{\Phi} \boldsymbol{\varphi} \boldsymbol{\psi}^{-2}$, and $D_{i}\left(\mathbf{A}_{\mathrm{y}}^{\prime}\right)=\boldsymbol{\Phi} D_{i}\left(\mathbf{A}_{\mathbf{y}}\right) \boldsymbol{\varphi} \boldsymbol{\psi}^{-2}=D_{i}\left(\mathbf{A}_{\mathbf{y}}\right) \boldsymbol{\eta}$. Then Eq. 2 is rewritten as

Define

$$
\mathbf{Y}=\left[\begin{array}{c}
\mathbf{A}_{\mathbf{x}}^{\prime} D_{1}\left(\mathbf{A}_{\mathbf{y}}\right) \\
\vdots \\
\mathbf{A}_{\mathbf{x}}^{\prime} D_{\mathbf{N}}\left(\mathbf{A}_{\mathbf{y}}\right) \\
\mathbf{A}_{\mathbf{x}}^{\prime} D_{1}\left(\mathbf{A}_{\mathbf{y}}^{\prime}\right) \\
\vdots \\
\mathbf{A}_{\mathbf{x}}^{\prime} D_{\mathbf{N}}\left(\mathbf{A}_{\mathbf{y}}^{\prime}\right)
\end{array}\right] \mathbf{S}_{0}+\tilde{\mathbf{N}}=\mathbf{A}_{1} \mathbf{S}_{0}+\tilde{\mathbf{N}}
$$

Then

$$
\mathbf{A}_{11}=\left[\begin{array}{c}
\mathbf{A}_{x}^{\prime} D_{1}\left(\mathbf{A}_{\mathbf{y}}\right) \\
\vdots \\
\mathbf{A}_{x}^{\prime} D_{N-1}\left(\mathbf{A}_{\mathbf{y}}\right) \\
\mathbf{A}_{x}^{\prime} D_{1}\left(\mathbf{A}_{\mathbf{y}}^{\prime}\right) \\
\vdots \\
\mathbf{A}_{x}^{\prime} D_{N-1}\left(\mathbf{A}_{\mathbf{y}}^{\prime}\right)
\end{array}\right], \mathbf{A}_{12}=\left[\begin{array}{c}
\mathbf{A}_{x}^{\prime} D_{2}\left(\mathbf{A}_{\mathbf{y}}\right) \\
\vdots \\
\mathbf{A}_{x}^{\prime} D_{N}\left(\mathbf{A}_{\mathbf{y}}\right) \\
\mathbf{A}_{x}^{\prime} D_{2}\left(\mathbf{A}_{\mathbf{y}}^{\prime}\right) \\
\vdots \\
\mathbf{A}_{x}^{\prime} D_{N}\left(\mathbf{A}_{\mathbf{y}}^{\prime}\right)
\end{array}\right]
$$

$$
\mathbf{A}_{12}=\mathbf{A}_{11} D_{2}\left(A_{y}\right)=\mathbf{A}_{11} \Psi_{y}
$$


Signal sub-space can be expressed as $E_{s}$. We define $E_{x 1}=\left[E_{s}(1: M(N-1),:) ; E_{s}(M N+1: 2 M N-M,:)\right]$ and $\left.E_{y 1}=\left[E_{s}(M+1: M N),:\right) ; E_{s}(M N+M+1: 2 M N,:)\right]$. Then

$$
\left[\begin{array}{c}
E_{x 1} \\
E_{y 1}
\end{array}\right]=\left[\begin{array}{c}
A_{11} T \\
A_{11} D_{2}\left(A_{y}\right) T
\end{array}\right]
$$

where $\mathrm{T}$ is full rank matrix of $K \times K$.

$$
E_{y 1}=E_{x 1} T^{-1} D_{2}\left(A_{y}\right) T=E_{x 1} T^{-1} \Psi_{y} T
$$

Through Least Square(LS) method we have

$$
\hat{\boldsymbol{\Psi}}_{y}=\mathbf{E}_{x 1}^{+} \mathbf{E}_{y 1}
$$

We obtain $\boldsymbol{\Psi}_{y}$ via eigenvalue decomposition of $\hat{\boldsymbol{\Psi}}_{y}$, then acquire

$$
\hat{r}_{y k}=\sin \hat{\phi}_{k} \sin \hat{\theta}_{k}, k=1,2, \cdots, K
$$

Reconstruct the signal sub-space, we obtain $\mathbf{E}_{s}{ }^{\prime}=\mathbf{E}_{s} \hat{\mathbf{T}}^{-1}$

Define

$$
\mathbf{E}_{s}^{\prime}=\left[\begin{array}{c}
\mathbf{A}_{y}^{\prime} D_{1}\left(\mathbf{A}_{x}\right) \\
\vdots \\
\mathbf{A}_{y}^{\prime} D_{N}\left(\mathbf{A}_{x}\right) \\
\mathbf{A}_{y}^{\prime} D_{1}\left(\mathbf{A}_{x}^{\prime}\right) \\
\vdots \\
\mathbf{A}_{y}^{\prime} D_{N}\left(\mathbf{A}_{x}^{\prime}\right)
\end{array}\right] \boldsymbol{\Pi}^{-1}
$$

$$
\begin{aligned}
& \mathbf{E}_{x 2}=\left[\mathbf{E}_{s}^{\prime}(1: N(M-1),:) ; \mathbf{E}_{s}{ }^{\prime}(M N+1: 2 M N-N,:)\right] \\
& \left.\mathbf{E}_{y 2}=\left[\mathbf{E}_{s}^{\prime}(N+1: M N),:\right) ; \mathbf{E}_{s}^{\prime}(M N+N+1: 2 M N,:)\right]
\end{aligned}
$$

Put

$$
\mathbf{A}_{3}=\left[\begin{array}{c}
\mathbf{A}_{y}^{\prime} D_{1}\left(\mathbf{A}_{x}\right) \\
\vdots \\
\mathbf{A}_{y}^{\prime} D_{N-1}\left(\mathbf{A}_{x}\right) \\
\mathbf{A}_{y}^{\prime} D_{1}\left(\mathbf{A}_{x}^{\prime}\right) \\
\vdots \\
\mathbf{A}_{y}^{\prime} D_{N-1}\left(\mathbf{A}_{x}^{\prime}\right)
\end{array}\right], \mathbf{A}_{4}=\left[\begin{array}{c}
\mathbf{A}_{y}^{\prime} D_{2}\left(\mathbf{A}_{x}\right) \\
\vdots \\
\mathbf{A}_{y}^{\prime} D_{N}\left(\mathbf{A}_{x}\right) \\
\mathbf{A}_{y}^{\prime} D_{2}\left(\mathbf{A}_{x}^{\prime}\right) \\
\vdots \\
\mathbf{A}_{y}^{\prime} D_{N}\left(\mathbf{A}_{x}^{\prime}\right)
\end{array}\right]
$$

Then

$$
\begin{aligned}
& \mathbf{E}_{x 2}=\mathbf{A}_{3} \boldsymbol{\Pi}^{-1} \\
& \mathbf{E}_{y 2}=\mathbf{A}_{3} \boldsymbol{\Phi}_{x} \boldsymbol{\Pi}^{-1}
\end{aligned}
$$

And we have

$$
\left(\mathbf{E}_{x 2}\right)^{+} \mathbf{E}_{y 2}=\boldsymbol{\Pi} \Phi_{x} \Pi^{-1}
$$

Without impact of noise, we get

$$
\hat{\mathbf{\Phi}}_{x}=\boldsymbol{\Pi} \boldsymbol{\Phi}_{x} \Pi^{-1}
$$

so far, we can obtain Eq.(15) by $\boldsymbol{\Phi}_{x}=\operatorname{diag}\left(e^{-j 2 \pi d \cos \theta_{1} \sin \phi_{1} / \lambda}, \ldots, e^{-j 2 \pi d \cos \theta_{K} \sin \phi_{\kappa} / \lambda}\right)$ 


$$
\hat{r}_{x k}=\cos \hat{\phi}_{k} \sin \hat{\theta}_{k}, k=1,2, \cdots, K
$$

Then, the 2D-DOA is estimated via

$$
\begin{aligned}
& \hat{\theta}_{k}=\arcsin \sqrt{\hat{r}_{x k}^{2}+\hat{r}_{y k}^{2}} \\
& \hat{\phi}_{k}=\arctan \sqrt{\hat{r}_{y k} / \hat{r}_{x k}}
\end{aligned}
$$

The complexity of the circular ESPRIT algorithm is $O\left(L M^{2} N^{2}+M^{3} N^{3}+2 K^{2}(M-1) N+3 K^{3}+\right.$ $\left.K^{2} M N+(N-1) M K\right)$, whereas that of the NC-ESPRIT is $O\left(4 L M^{2} N^{2}+8 M^{3} N^{3}+4 K^{2}(M-1) N+\right.$ $\left.3 K^{3}+2 K^{2} M N+2(N-1) M K\right)$. Apparently, extending the received data matrix by exploiting the property of noncircular signals brings the doubled array aperture and better 2D-DOA estimation performance, as well as heavier computational load than the circular ESPRIT algorithm.

\section{Simulation results}

Define the root mean squared error (RMSE) as

$$
R M S E=\frac{1}{K} \sum_{k=1}^{K} \sqrt{\frac{1}{I} \sum_{i=1}^{I}\left(\hat{a}_{k, i}-a_{k}\right)^{2}}
$$

where $\hat{a}_{k, i}$ is the estimate of elevation angle/azimuth angle $a_{k}$ of the ith Monte Carlo trial and $I$ represents the number of total Monte Carlo trials during each simulation. Assume that there are three uncorrelated sources $(K=3)$ with arriving angles $\left(\phi_{1}, \theta_{1}\right)=\left(15^{\circ}, 10^{\circ}\right),\left(\phi_{2}, \theta_{2}\right)=\left(25^{\circ}, 20^{\circ}\right)$, and $\left(\phi_{3}, \theta_{3}\right)=\left(35^{\circ}, 30^{\circ}\right)$, respectively. The receive array is a uniform rectangular array with the antenna spacing of half wavelength.

The 2D-DOA estimation result of our algorithm with signal-to-noise ratio (SNR) $=0 \mathrm{~dB}$ and $15 \mathrm{~dB}$ is shown in Fig. 1 and Fig. 2( $M=8, N=8, L=100)$, respectively. Simulation results indicate the effectiveness of our algorithm. Figs. 3 present the angle estimation performance comparisons among the proposed NC-ESPRIT algorithm, the conventional ESPRIT algorithm [7] and the $\operatorname{CRB}(M=8, N=8, L=100, K=3)$. From Fig. 3, it can be seen that by exploiting the property of noncircular signals to double the array aperture, the proposed NC-ESPRIT algorithm has better angle estimation than the conventional ESPRIT algorithm. Fig.4 depicts the angle estimation performance of the proposed algorithm with different numbers of snapshots $L$ ( $M=8, N=8, K=3$ ). As the figure shows, the angle estimation performance of the proposed algorithm is significantly improved when $L$ increases.

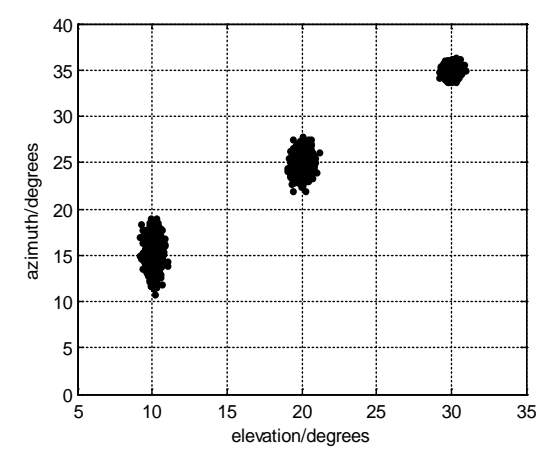

Fig. 1 Estimation results with $\mathrm{SNR}=0 \mathrm{~dB}$

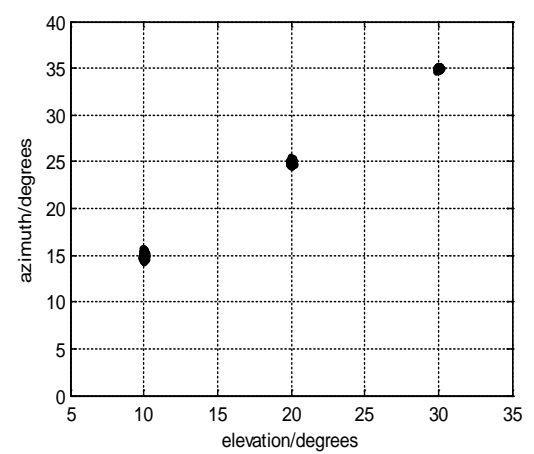

Fig. 2 Estimation results with SNR=15dB 

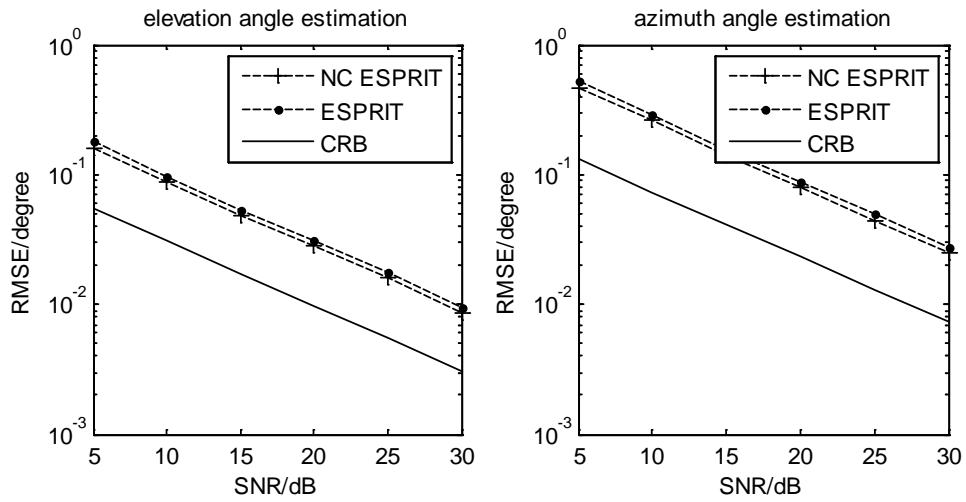

Fig. 3 The angle estimation performance comparison
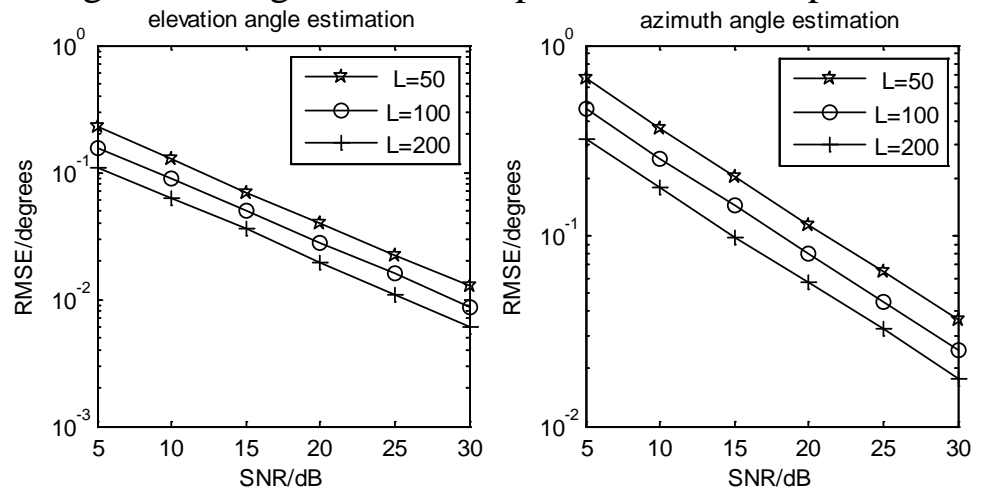

Fig. 4 Angle estimation performance with different $L$

\section{Conclusions}

In this paper, we address the problem of 2D-DOA estimation for noncircular signals with uniform rectangular array, and propose a NC-ESPRIT algorithm. Based on the conventional ESPRIT algorithm, we utilize the noncircularity of the signals and extend the receive data matrix to double the array aperture. The proposed algorithm has better 2D-DOA estimation performance than the ESPRIT algorithm. Moreover, our algorithm can achieve automatically paired 2D-DOA estimation and does not require spectral peak searching.

\section{References}

[1] Krim, H., and M. Viberg: Two decades of array signal processing research: the parametric approach. Signal Processing Magazine IEEE13.4(1996):67 - 94.

[2] Thng, I., A. Cantoni, and Y. H. Leung: Derivative constrained optimum broad-band antenna arrays. IEEE Transactions on Signal Processing41.7(1993):2376-2388.

[3] Doğan, Mithat C., and Mendel, Jeny M.: Applications of cumulants to array processing .I. Aperture extension and array calibration. IEEE Transactions on Signal Processing 43.5(1995):1200-1216.

[4] Zhang, Xiaofei, et al.: A Novel DOA estimation Algorithm Based on Eigen Space. Microwave, Antenna, Propagation and EMC Technologies for Wireless Communications, 2007 International Symposium on IEEE, 2007:551-554.

[5] Delmas, Jean Pierre, and H. Abeida: Stochastic Cramér-Rao bound for noncircular signals with application to DOA estimation. Signal Processing IEEE Transactions on 52.11(2004):3192-3199.

[6] Schreier, Peter J., and L. L. Scharf. Statistical signal processing of complex-valued data: the theory of improper and noncircular signals. Cambridge University Press, (2010). 
[7] Haardt M, Römer F. Enhancements of unitary ESPRIT for noncircular sources, in Proceedings of 29th IEEE International Conference on Acoustics, Speech, and Signal Processing, Montreal, Quebec, Canada, May 17-21, (2004), pp. II-101-II-104.

[8] A. Zoubir, P. Charg'e, and Y. Wang: Non circular sources localization with ESPRIT, in Proc. European Conference on Wireless Technology (ECWT 2003), Munich, Germany, Oct.(2003).

[9] Roemer, Florian, and M. Haardt: Efficient 1-D and 2-D DOA Estimation for Non-Circular Sourceswith Hexagonal Shaped Espar Arrays. Acoustics Speech \& Signal Processing .icassp Proceedings.ieee International Conf 4(2006):IV. 